\title{
Disseminated cryptococcosis in a patient receiving chronic haemodialysis
}

\author{
Richard Banks, Adrian Williams, Stuart Glover ${ }^{1}$, Patricia Burton, David Warnock ${ }^{2}$ \\ and Campbell Mackenzie
}

Department of Nephrology and Histopathology, Southmead Hospital, ${ }^{\prime}$ Department of Infectious Diseases, Ham Green Hospital, and ${ }^{2}$ Department of Microbiology, Bristol Royal Infirmary, Bristol, UK.

\begin{abstract}
Summary: Systemic infection with Cryptococcosis neoformans is described in a young man receiving maintenance haemodialysis. The organism was identified in thin sections of a cervical lymph node using resin embedding and silver staining. There were no predisposing factors other than uraemia. His clinical infection responded to the combination of oral 5-flucytosine and a total of $2.5 \mathrm{~g}$ of intravenous amphotericin $B$. He remains free of relapse after 30 months.
\end{abstract}

\section{Introduction}

Cryptococcosis is a sub-acute or chronic infection caused by the yeast Cryptococcus neoformans. The organism is widely distributed in soils and is frequently found in pigeon guano. Human infection occurs mainly via the respiratory tract and in immunocompromised patients (Hay, 1982). Despite the ubiquity of the organism and the reduced immune tolerance associated with chronic renal failure (Touraine et al., 1975), we are unaware of a previous report of systemic cryptococcosis in a patient receiving maintenance haemodialysis.

\section{Case report}

A 22 year old male geography student with chronic renal failure secondary to glomerulonephritis, well controlled on haemodialysis for $2 \mathrm{y}$, presented with a swinging fever, dry cough and malaise. Eighteen months before, a cadaveric renal transplant was removed after 2 weeks following secondary haemorrhage, from which recovery was complete. One month before presentation the patient had been on a field course handling soil samples. On examination his oral temperature was $40.1^{\circ} \mathrm{C}$ and cervical lymphadenopathy was present. There was no clinical evidence of meningitis. White cell count was $7.1 \times 10^{9} / 1$, with $18 \%$ lymphocytes. Viral serology, blood, sputum, throat and urine cultures were all negative. Chest $\mathrm{X}$ -

Correspondence: R.A. Banks, B.Sc., M.D., M.R.C.P., Department of Renal Medicine, Southmead Hospital, Bristol BS10 5NB, UK.

Accepted: 7 February 1985. ray demonstrated miliary mottling in both lung fields with hilar enlargement and a small left pleural effusion. An initial diagnosis of tuberculosis was made and antituberculous therapy instituted. Over the following week the pyrexia remained and progressive hepatosplenomegaly was noted together with a large pericardial effusion, the latter confirmed by echocardiography. Biopsy of a cervical lymph node showed caseating tuberculoid lymph granulomata with a few pale, encapsulated, spherical organisms which, on staining with the Gomori methenamine silver method were suggestive of $C$. neoformans. Resin embedded sections stained by the same technique greatly improved resolution and revealed many budding, encapsulated and intracellular organisms typical of cryptococci (Figure 1). The diagnosis of cryptococcosis was confirmed by a serum antibody titre of $1: 4$. Tests for cryptococcal antigen were negative.

His treatment consisted of amphotericin B on alternate days, increasing to $0.6 \mathrm{mg} / \mathrm{kg}$ and infused during haemodialysis, together with oral 5-flucytosine $50 \mathrm{mg} / \mathrm{kg}$ after each haemodialysis. Blood levels of the latter were measured at intervals. Within $24 \mathrm{~h}$ he was apyrexial. Two weeks later the hepatosplenomegaly had markedly regressed and the pericardial effusion completely resolved, but a sinus which had formed after the lymph node biopsy continued to discharge pus. Culture of pus, lymph node, urine and blood in nitrogen enriched media failed to grow $C$. neoformans or other fungi. After 6 weeks and a total dose of $1.5 \mathrm{~g}$ of amphotericin B he was discharged only to return $6 \mathrm{~d}$ later with enlarging lymphadenopathy and malaise. Therapy was recommenced and resulted in rapid clinical improvement. After a further 4 weeks he had 

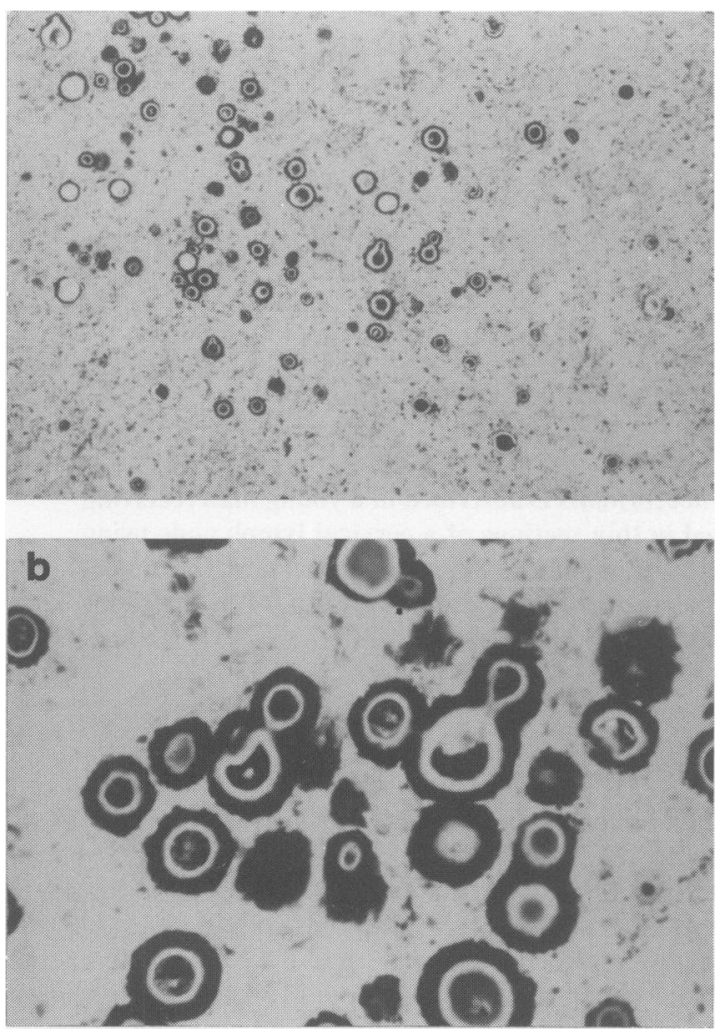

Figure I Photomicrographs of cervical lymph node biopsy showing encapsulated budding cryptococci. Resin embedded, thin sections stained by the Gomori methenamine silver method (a) $\times 480$; (b) $\times 1920$.

received a total dose of $2.5 \mathrm{~g}$ of amphotericin B. Five weeks after discharge serum cryptococcal antibody titre was negative, and after 30 months he remains well with complete resolution of his hepatosplenomegaly and lymphadenopathy, chest X-ray and serology.

\section{Discussion}

Disseminated cryptococcosis developing in a patient on maintenance haemodialysis has not previously been reported, even though other mycotic infections (e.g. coccidioidomycosis) are common in dialysis units situated in endemic areas outside the UK (Cohen $e t$ al., 1982). Our patient may have inhaled spores from soil samples but he was not predisposed to systemic infection other than by virtue of uraemia which is known to reduce cell-mediated immunity (Touraine $e t$ al., 1975), and impair neutrophil chemotaxis (Lane et al., 1966). He had not received any immuno-suppressive drugs except for 2 weeks after renal transplantation, 18 months before presentation.

The incidence of cryptococcosis in the general population is difficult to assess. There are 8-13 cases annually in the UK, $85 \%$ of which occur in predisposed patients (Hay et al., 1980). However, the organism is difficult to culture and diagnosis often depends on serology or histopathology. This latter provides a rapid means of diagnosis and the present case report suggests that resin embedding with silver staining may increase the sensitivity of this approach.

The combination of amphotericin B and 5flucytosine is synergistic in the treatment of cryptococcosis, allowing for a reduction of these potentially toxic agents (Utz et al., 1975). Nephrotoxicity often limits the use of amphotericin B but was irrelevant in our patient on maintenance haemodialysis. In contrast, the phlebitis associated with this drug may have jeopardized future vascular access. We elected to administer amphotericin $\mathbf{B}$ on alternate days on dialysis in anticipation that the high blood flow through the artificial kidney would minimize risk to his arteriovenous fistula. He received his amphotericin $B$ in this fashion for a total of 10 weeks without adverse effect to the vessels.

The optimal duration of anticryptococcal therapy is difficult to ascertain. Transformation of the serum antigen titre from positive to negative is often taken as evidence of cure (Hay, 1982), as is reduction of white cell count in the cerebrospinal fluid. Cryptococcal antigen titre was negative in our patient's serum before therapy and this somewhat surprising finding may have been related to his uraemia. At no time did our patient have signs of meningeal involvement and lumbar puncture was, therefore, deemed unwarranted. In the absence of meningitis the duration of anticryptococcal therapy is often reduced but the rapid relapse after the first course of chemotherapy in our patient and the prolonged remission after a total dose of $2.5 \mathrm{~g}$ of amphotericin B, supports the observation that an extended course of therapy is necessary in the treatment of disseminated cryptococcosis.

\section{Acknowledgements}

We are grateful to Miss K. Edmonds for typing this report and to Dr D. Reeves and staff of the Department of Microbiology, Southmead General Hospital, for their assistance during the management of this patient, and criticism of the text. 


\section{References}

COHEN, I.M., GALGIANI. J.N., POTTER D. \& OGDEN, A (1982). Coccidioidomycosis in renal replacement therapy. Archives of Internal Medicine, 142, 489.

HAY, R.J. (1982). Clinical manifestations and management of cryptococcosis in the compromised patient. In Fungal Infection in the Compromised Patient, Warnock, D.W., Richards, M.D. (Eds): p. 39, John Wiley and Sons: Chichester.

HAY, R.J., MACKENZIE, D.W.R., CAMPBELL, C.K. \& PHILPOT, C.M. (1980). Cryptococcosis in the United Kingdom and the Irish Republic; an analysis of 69 cases. Journal of Infection, 2, 13.
LANE, P., RITZMANN, S. \& MERIAN, F. (1966). Cellular evolution in induced inflammation in uraemic patients. Texas Reports on Biology in Medicine, 24, 107.

TOURAINE, J.L., TOURAINE, J.P. \& REVILLARD, J. (1975). T-lymphocyte and serum inhibitors of cell-mediated immunity in renal insufficiency. Nephron, 14, 195.

UTZ, J.P., GARRIQUES, I.L. \& SANDE, M.A. (1975). Therapy of cryptococcosis with a combination of flucytosine and amphotericin B. Journal of Infectious Diseases, 132, 368. 